\title{
Clinical effects of carvedilol and trimetazidine for the treatmentof alcoholic myocardiopathy
}

\author{
HUI LI, FU-YUAN LIU, XIAO-LAN LI, XIAO-MEI LI and LEI ZHU \\ Department of Cardiovascularology, Xiangyang Hospital Affiliated to Hubei University of Medicine, \\ Xiangyang, Hubei 441001, P.R. China
}

Received March 4, 2016; Accepted May 19, 2016

DOI: $10.3892 /$ etm.2016.3363

\begin{abstract}
The aim of the study was to compare the clinical effects of carvedilol and trimetazidine for the treatment of alcoholic cardiomyopathy. A total of 60 patients diagnosed with alcoholic cardiomyopathy were enrolled in the study. The patients were randomly divided into the carvedilol $(n=20)$, trimetazidine $(n=20)$ and control $(n=20)$ groups. The patients in the control, carvedilol and trimetazidine groups were treated with conventional drugs, conventional drugs + carvedil and conventional drugs + trimetazidine respectively, for 12 weeks. The patients were compared for their heart functions [left ventricular ejection fraction (LVEF), C-reactive protein (CRP) and 6 min walking], heart rate, blood pressure and heart enlargement (cardiothoracic proportion and left ventricular diameter) before and after treatment. The parameters studied for heart functions, heart rate, blood pressure, heart enlargement, clinical effects before and after treatment were statistically insignificant $(\mathrm{p}>0.05)$. After treatment, the carvedilol and trimetazidine groups showed higher LVEF and $\mathrm{CRP}$, longer walking distance in $6 \mathrm{~min}$, as well as lower heart rate and blood pressure (both systolic and diastolic) compared to the control group. Similarly, the cardiothoracic proportion and left ventricular internal diameter for the carvedilol and trimetazidine groups was lower than those of the control group, with better clinical effects $(\mathrm{p}<0.05)$. In conclusion, the curative effects of the carvedilol and trimetazidine groups of alcoholic myocardiopathy similar. Both are safe agents that may improve the cardiac function and heart expansion of patients.
\end{abstract}

\section{Introduction}

Alcoholic cardiomyopathy is a type of myocardial degeneration resulting from a large amount of alcohol consumption with varied clinical manifestations such as cardiac enlargement,

Correspondence to: Dr Fu-Yuan Liu, Department of Cardiovascularology, Xiangyang Hospital Affiliated to Hubei University of Medicine, 15 Jiefang Road, Xiangyang, Hubei 441000, P.R. China E-mail:1fy0053@163.com

Key words: carvedilol, trimetazidine, alcoholic myocardiopathy cardiac insufficiency and arrhythmia, which mainly occurs in the 30- to 50-year age group $(1,2)$. In recent years, with improvement of the standard of living and changes of lifestyle, the number of heavy drinking in China has been increasing rapidly, which is inevitably leading to enhanced risks of alcoholic cardiomyopathy (3). Patients with relatively milder symptoms may gradually recover following discontinuation of alcohol consumption.

In the present study, 60 patients with alcoholic cardiomyopathy at the Department of Cardiovascularology, Xiangyang Hospital (Hubei, China) were selected and the clinical effects of the treatment of carvedilol and trimetazidine were compared.

\section{Patients and methods}

Patients. Sixty patients, diagnosed with alcoholic cardiomyopathy at the Department of Cardiovascularology, Xiangyang Hospital were selected between March 2012 and July 2014. The patients conformed to the clinical diagnostic criteria. The patients were randomly divided the patients into the carvedilol $(n=20)$, trimetazidine $(n=20)$ and control $(n=20)$ groups. The carvedilol group included 17 men and 3 women with an age range of 31-64 years and an average age of $45.8 \pm 4.6$ years. Alcohol consumption for this group was 8.1-18.6 years with an average of $12.2 \pm 3.9$ years, and the cardiac functional grading for 13 and 7 cases was grade II and III, respectively. The trimetazidine group included 16 men and 4 women with an age range of 30-65 years and an average of $46.8 \pm 4.2$ years. Alcohol consumption for this group was 7.8-19.0 years with an average of $11.9 \pm 3.6$ years, and the cardiac functional grading for 14 and 6 cases was grade II and III, respectively. The control group included 18 men and 2 women with an age range of 33-64 years and an average of 46.6 \pm 4.3 years. Alcohol consumption for this group was $746.6 \pm 4.3$ years with an average of $11.3 \pm 3.3$ years, and cardiac functional grading for 15 and 5 cases was grade II and III level, respectively. Differences in gender, age, alcohol consumption years and cardiac functional grading of the three groups were not statistically significant $(\mathrm{p}>0.05)$.

Inclusion and exclusion criteria. Inclusion criteria for the study were: i) History of long-term ( $\geq 6$ years) heavy consumption of alcohol or bibulosity; ii) heart failure and cardiac enlargement and other symptoms; iii) amount of alcohold consumed for 
Table I. Comparison of cardiac function indexes before and after treatment (mean $\pm \mathrm{SD}$ ).

\begin{tabular}{|c|c|c|c|c|c|c|c|}
\hline \multirow[b]{2}{*}{ Group } & \multirow[b]{2}{*}{ Case } & \multicolumn{2}{|c|}{$\operatorname{LVEF}(\%)$} & \multicolumn{2}{|c|}{$\mathrm{CRP}(\mathrm{mg} / \mathrm{l})$} & \multicolumn{2}{|c|}{ 6-min walking distance $(\mathrm{m})$} \\
\hline & & Pre-treatment & Post-treatment & Pre-treatment & Post-treatment & Pre-treatment & Post-treatment \\
\hline Control & 20 & $37.4 \pm 8.2$ & $41.4 \pm 7.9$ & $12.3 \pm 1.3$ & $8.8 \pm 0.9$ & $300.7 \pm 46.3$ & $474.8 \pm 58.2$ \\
\hline Carvedilol & 20 & $36.9 \pm 9.1$ & $44.8 \pm 7.9^{\mathrm{a}}$ & $12.3 \pm 1.3$ & $5.1 \pm 0.6^{\mathrm{a}}$ & $298.9 \pm 45.1$ & $540.7 \pm 48.2^{\mathrm{a}}$ \\
\hline Trimetazidine & 20 & $36.8 \pm 8.5$ & $45.2 \pm 8.2^{\mathrm{a}}$ & $12.3 \pm 1.3$ & $5.3 \pm 0.7^{\mathrm{a}}$ & $298.9 \pm 45.1$ & $546.4 \pm 69.4^{\mathrm{a}}$ \\
\hline $\mathrm{F}$ & & 0.642 & 3.714 & 0.000 & 6.732 & 1.246 & 11.484 \\
\hline P-value & & $>0.05$ & $<0.05$ & $>0.05$ & $<0.05$ & $>0.05$ & $<0.05$ \\
\hline
\end{tabular}

LVEF, left ventricular ejection fraction; CRP, C-reactive protein. Compared with the control group, ${ }^{\mathrm{a}} \mathrm{P}<0.05$.

men was $>80 \mathrm{~g} / \mathrm{day}$, and women was $>40 \mathrm{~g} /$ day; and iv) agreed with the study and provided written informed consent.

Exclusion criteria for the study were: i) Patients with other heart diseases; ii) patients who disagreed with the study, or had a drinking temperance $<6$ months; and iii) patients with incomplete clinical data.

The present study was approved by the Ethics Committee of Xiangyang Hospital.

Treatment methods. The patients in the control group were administered conventional drugs, including digoxin, diuretics, angiotensin-converting enzyme inhibitor (ACEI) and spironolactone, and cardiac color ultrasound and chest X-ray examinations were performed. The changes of pulmonary rates, blood pressure and heart rate were observed daily, electrocardiographic examination was performed once every three days and the patients were suggested to discontinue consumption of alcohol and take a good rest.

The patients in the carvedilol group were administered conventional drugs and carvedilol, initial dose of $6.25 \mathrm{mg} / \mathrm{time}$, twice per day orally. If patients tolerated the dose, the systolic blood pressure was measured in erect position $1 \mathrm{~h}$ following drug treatment, and the dose was maintained for 7-14 days, which was subsequently increased to $12.5 \mathrm{mg} /$ time, twice per day orally.

For the trimetazidine group, the patients were administered with conventional drugs and trimetazidin. The dosage regimen was $20 \mathrm{mg} /$ time, three times per day after a meal.

Observation indices. The heart functions [left ventricular ejection fraction (LVEF), C-reactive protein (CRP), and 6-min walking distance], heart rate, blood pressure (systolic pressure and diastolic pressure) and heart enlargement (cardiothoracic proportion and left ventricular diameter) were compared for the three groups prior to and after treatment.

Curative effects assessment standard. Excellence was considered when cardiac function was improved by $\geq 2$ levels. Effective was considered when cardiac function was improved by 1 level. Ineffective was considered when cardiac function was not improved or even deteriorated.

Statistical analysis. SPSS 15.0 statistical software (SPSS 15.0 statistical software (SPSS Inc., Chicago, IL, USA) was used for statistical analysis. Data were presented as mean \pm standard deviation. The comparison among groups was made by variance analysis. The pair-wise comparison was made by using the q-test. Enumeration data were tested by using the $\chi^{2}$ test, ranked data were tested using the rank-sum test. Difference was of statistical significance in case of the bilateral $\mathrm{p}<0.05$.

\section{Results}

Comparison of cardiac function indices prior to and after treatment. Prior to treatment, LVEF, CRP, and 6-min walking distance of the three groups were not statistically different ( $p>0.05$ ). Following treatment, the differences of LVEF, CRP, and 6-min walking distance of the three groups were statistically different $(\mathrm{p}<0.05)$. In comparison to the control group, the carvedilol and trimetazidine groups showed significant improvement in LVEF and CRP and the 6-min walking distance $(p<0.05)$. However, the differences between the carvedilol and trimetazidine groups were not statistically significant ( $\mathrm{p}>0.05)$ (Table I).

Comparison of heart rate and blood pressure prior to and after treatment. Prior to treatment, the heart rate, blood pressure (systolic and diastolic) of the three groups were not statistically different $(\mathrm{p}>0.05)$. Following treatment, the differences of heart rate, blood pressure (systolic and diastolic) of the three groups were statistically different $(\mathrm{p}<0.05)$. The heart rate, blood pressure (systolic and diastolic) of the carvedilol and trimetazidine groups were significantly lower than those of the control groups $(\mathrm{p}<0.05)$. However, differences between the carvedilol and trimetazidine groups were not statistically significant ( $\mathrm{p}>0.05)$ (Table II).

Comparison of cardiac enlargement indices prior to and after treatment. Prior to treatment, the cardiothoracic proportion and left ventricular internal diameter of the three groups were not statistically different $(\mathrm{p}>0.05)$. Following treatment, differences of cardiothoracic proportion and left ventricular internal diameter of the three groups were statistically different $(\mathrm{p}<0.05)$. The cardiothoracic proportion and left ventricular internal diameter of the carvedilol and trimetazidine groups were significantly higher than those of the control group $(\mathrm{p}<0.05)$. However, the differences between the carvedilol 
Table II. Comparison of the heart rate and blood pressure prior to and after treatment (mean \pm SD).

\begin{tabular}{|c|c|c|c|c|c|c|c|}
\hline \multirow[b]{2}{*}{ Group } & \multirow[b]{2}{*}{ Case } & \multicolumn{2}{|c|}{ Heart rate (times/min) } & \multicolumn{2}{|c|}{ Systolic pressure (mmHg) } & \multicolumn{2}{|c|}{ Diastolic pressure $(\mathrm{mmHg})$} \\
\hline & & Pre-treatment & Post-treatment & Pre-treatment & Post-treatment & Pre-treatment & Post-treatment \\
\hline Control & 20 & $90 \pm 19$ & $86 \pm 18$ & $147 \pm 27$ & $137 \pm 69$ & $79 \pm 11$ & $74 \pm 10$ \\
\hline Carvedilol & 20 & $89 \pm 18$ & $76 \pm 7^{\mathrm{a}}$ & $149 \pm 26$ & $122 \pm 71^{\mathrm{a}}$ & $78 \pm 10$ & $68 \pm 11^{\mathrm{a}}$ \\
\hline Trimetazidine & 20 & $91 \pm 18$ & $75 \pm 8^{\mathrm{a}}$ & $146 \pm 27$ & $120 \pm 71^{\mathrm{a}}$ & $80 \pm 12$ & $65 \pm 10^{\mathrm{a}}$ \\
\hline $\mathrm{F}$ & & 0.843 & 7.241 & 0.116 & 5.384 & 0.942 & 4.926 \\
\hline P-value & & $>0.05$ & $<0.05$ & $>0.05$ & $<0.05$ & $>0.05$ & $<0.05$ \\
\hline
\end{tabular}

$1 \mathrm{mmHg}=0.133 \mathrm{kPa}$; compared with the control group: ${ }^{\mathrm{a}} \mathrm{p}<0.05$.

Table III. Comparison of cardiac enlargement indices prior to and after treatment (mean \pm SD).

\begin{tabular}{|c|c|c|c|c|c|}
\hline \multirow[b]{2}{*}{ Group } & \multirow[b]{2}{*}{ Case } & \multicolumn{2}{|c|}{ CTR (\%) } & \multicolumn{2}{|c|}{ LVID (\%) } \\
\hline & & Pre-treatment & Post-treatment & Pre-treatment & Post-treatment \\
\hline Control & 20 & $0.68 \pm 0.11$ & $0.64 \pm 0.10$ & $58.8 \pm 0.7$ & $52.6 \pm 0.5$ \\
\hline Carvedilol & 20 & $0.66 \pm 0.09$ & $0.55 \pm 0.12$ & $59.7 \pm 0.6$ & $46.5 \pm 0.8^{a}$ \\
\hline Trimetazidine & 20 & $0.71 \pm 0.13$ & $0.51 \pm 0.08$ & $59.2 \pm 0.7$ & $45.8 \pm 0.9^{\mathrm{a}}$ \\
\hline $\mathrm{F}$ & & 0.347 & 3.972 & 0.142 & 4.286 \\
\hline P-value & & $>0.05$ & $<0.05$ & $>0.05$ & $<0.05$ \\
\hline
\end{tabular}

CTR, cardiothoracic. LVID, left ventricular internal diameter. Compared with the control group, ${ }^{a} \mathrm{p}<0.05$.

Table IV. Comparison of the clinical effects in different groups.

\begin{tabular}{lcccc}
\hline Group & Case & Excellence & Effective & Ineffective \\
\hline Control & 20 & 4 & 8 & 8 \\
Carvedilol & 20 & 11 & 7 & 2 \\
Trimetazidine & 20 & 12 & 7 & 1 \\
\hline
\end{tabular}

and trimetazidine groups were not statistically significant ( $>0.05)$ (Table III).

Comparison of clinical effects. Differences of clinical effects of the three groups were statistically different. The clinical effects of the carvedilol and trimetazidine groups were significantly improved compared to the control group $(\mathrm{p}<0.05)$. On the other hand, the differences between the carvedilol and trimetazidine groups were not statistically significant $(\mathrm{p}>0.05)$ (Table IV). In addition, there were no obvious adverse reactions in any of the three groups.

\section{Discussion}

Alcoholic myocardiopathy is a type of non-ischemic dilated cardiomyopathy caused by long-term alcohol consumption (4). The mechanisms by which ethanol can induce cardiomyopathy include destruction of the integrity of myocardial cell membrane and effects on ion exchange and information transfer of various cardiac muscle cells; functional change of mitochondria and other organelles, leading to shortage of myocardial energy; abnormal fat deposition and changes of activity of ATP and inhibition of myocardial activity; imbalance of nutrition and aggravation of myocardial damages (5-7). In addition, ethanol can also seriously affect the permeability of ions, leading to loss of potassium phosphate and calcium overload, which further inhibits the contraction and relaxation of myocardium. The clinical manifestations of alcoholic cardiomyopathy are diverse, mainly including arrhythmia, heart failure and cardiac insufficiency. Alcoholic myocardiopathy mainly occurs in men and accounts for $90 \%$ (8). The study by Schwarz et al (9) showed that alcoholic cardiomyopathy accounted for approximately $1 / 3$ of dilated cardiomyopathy.

Patients with relatively milder symptoms may gradually recover following discontinuation of alcohol consumption. However, a patient with severe symptoms should accept drug treatment. Common drugs include diuretics, digitalis, angiectasis drugs, ACEI, $\beta$-blockers, hypokalemia and hypomagnesemia $(10,11)$. Carvedilol is a $\beta$-blocker that may block the receptors and relax vessels. Trimetazidine is an anti-ischemic drug used for optimizing myocardial energy metabolism, which may affect the metabolism of myocardial cells, improve tissue tolerance to hypoxia and eliminate oxygen-free radicals (12). In the present study, carvedilol and trimetazidine were used to treat alcoholic myocardiopathy. The results strongly suggest the benefits of carvedilol and trimetazidine for the treatment of alcohol-induced myocardiopathy. In comparison to the control groups, the treated groups showed significantly higher LVEF and 
CRP, farther 6-min walking distance, lower heart rate and blood pressure (systolic and diastolic), lower cardiothoracic proportion and left ventricular internal diameter, along with better clinical effects. A comparison of the above indicators between the carvedilol and trimetazidine groups revealed that differences were statistically non-significant, indicating that the curative effects of carvedilol and trimetazidine for the treatment of alcoholic myocardiopathy were similar. The results were consistent with earlier studies of Duan et al (13) and Dijkstra et al (14), indicating that carvedilol and trimetazidine were capable of improving cardiac function and heart expansion without associated risk.

\section{References}

1. Kollar J: [Clinical picture of alcoholic myocardiopathy. Review]. Vnitr Lek 18: 172-181, 1972.

2. Mamas MA, Deaton C, Rutter MK, Yuille M, Williams SG, Ray SG, New J, Gibson JM and Neyses L: Impaired glucose tolerance and insulin resistance in heart failure: underrecognized and undertreated? J Card Fail 16: 761-768, 2010.

3. Jun $\mathrm{H}$ and Bo Y: New progress in the pathogenesis of alcoholic cardiomyopathy. Chin J Evid-Based Cardiovasc Med 6: 236-237, 2014 (In Chinese).

4. Estruch RR: [Alcoholic myocardiopathy]. Rev Clin Esp 201: 137-139, 2001.

5. Sugihara K, Fujimoto S, Motomiya Y, Hashimoto T, Nakamura S and Dohi K: Usefulness of long axis M-mode echocardiographic measurements for optimum dialysis in patients on maintenance hemodialysis: comparison with changes in plasma levels of atrial natriuretic peptide and brain natriuretic peptide. Clin Nephrol 56: 140-149, 2001
6. Gvozdjak A, Bada V, Kruty F, Niderland TR and Gvozdjak J: [Mechanism of the development of cardiac insufficiency in alcoholic myocardiopathy]. Kardiologiia 16: 136-137, 1976.

7. Mahfoudh-Boussaid A, Hadj Ayed Tka K, Zaouali MA, Roselló-Catafau J and Ben Abdennebi H: Effects of trimetazidine on the Akt/eNOS signaling pathway and oxidative stress in an in vivo rat model of renal ischemia-reperfusion. Ren Fail 36: 1436-1442, 2014.

8. Wu H, Yang J and Ding J: Effects of capsule on cardiac functions and expression of HMGB1 of patients with dilated cardiomyopathy. Chin J Evid-Based Cardiovasc Med 6: 271-273, 2014 (In Chinese).

9. Schwarz F, Mall G, Zebe H, Schmitzer E, Manthey J, Scheurlen H and Kübler W: Determinants of survival in patients with congestive cardiomyopathy: quantitative morphologic findings and left ventricular hemodynamics. Circulation 70: 923-928, 1984.

10. Arranz CJ, Lopez JL, Moya MM and Martin JT: [Reversibility of alcoholic myocardiopathy]. Med Clin (Barc) 93: 316-317, 1989.

11. Wang CM, Almsherqi ZA, McLachlan CS, Matthews S, Ramachandran M, Tay SK and Deng Y: Acute starvation in C57BL/6J mice increases myocardial UCP2 and UCP3 protein expression levels and decreases mitochondrial bio-energetic function. Stress 14: 66-72, 2011.

12. Aubert A, Bernard C, Clauser P, Harpey C and Vaudry H: [A cellular anti-ischemic agent, trimetazidine prevents the deleterious effects of oxygen free-radicals on the internal ear]. Ann Otolaryngol Chir Cervicofac 107: 28-35 1990 (In French).

13. Duan M, Wang L, Cao X and Huang G: The effect of coenzyme Q10 combined with trimetazidine for treatment of elderly patients with alcoholic cardiomyopathy heart failure. Chin J Gerontol 32: 4379-4381, 2012.

14. Dijkstra AF, Verdonk P and Lagro-Janssen AL: Gender bias in medical textbooks: examples from coronary heart disease, depression, alcohol abuse and pharmacology. Med Educ 42: 1021-1028, 2008 